\title{
The Relationship between the Zimbardo Time Perspective Inventory (ZTPI) and the Hofstede-Dimensions: Preliminary Empirical Evidence from Germany, Morocco, and China
}

\author{
Alexander Unger ${ }^{1}$, Julie Papastamatelou', Karim Gassemi², Alan Lu ${ }^{3}$ \\ ${ }^{1}$ University of Applied Sciences, Ludwigshafen, Germany \\ ${ }^{2}$ Ecole Nationale de Commerce et de Gestion de Casablanca, Université de Hassan II, Morocco \\ ${ }^{3}$ School of Management, Fuzhou University, Fuzhou, China \\ Email: alunger@web.de, JPapas@gmx.net, gassemik@gmail.com, alanlu0510@sina.com
}

Received January 2014

\begin{abstract}
In this study we intend to test possible relationships between time perspectives and the Hofstede dimensions at the individual level in three cultural settings: Germany, Morocco, and China. The main basic assumption is that the configuration of time perspectives [1] influences the cultural dimensions at the individual level. We assume that time perspectives are relevant for some of the cultural dimensions, and we tested these hypotheses separately in the three cultural settings used (Germany, Morocco and China).
\end{abstract}

\section{Keywords}

Zimbardo Time Persepctive Inventory, Hofstede Cultural Dimensions, Cross-Cultural Differences

\section{Zimbardo Time Perspective Inventory (ZTPI)}

Numerous empirical studies have shown that the concept of time perspectives developed by [1] has essential influence on many facets of human behavior. According to the theory, five independent dimensions were assumed: a) Positive Past (PP), b) Negative Past (NP), c) Present Hedonistic (PH), d) Present Fatalistic (PF), and e) Future (F). A sixth dimension was developed separately, which is named Transcendental Future [2] and refers to assumption according to the time after death (highly influenced by religious beliefs or philosophical orientations). This sixth dimension is not considered in the current study. For each of the dimension, it is assumed that each individual could score independently low or high. For each dimension, a mean index is calculated ranging from 1 (indicating lowest possible scoring) to 5 (indicating highest possible scoring). PP is characterized by a positive view of one's past, whereas NP in contrast is characterized by a negative view of one's past. Individuals scoring 
high on PH show a strong orientation toward immediate gratification and pleasure seeking. In turn, these individuals show typically low self-control-e.g., in persistence in the context of goal striving. Individuals scoring high on PF believe that their successes or failures in life did not depend on their own efforts but on faith, other persons, or in general on factors from outside on which they do not have any influence at all. A high score at the F dimension implies high levels of self-control and high working achievement. High F orientation is highly beneficial for success in life and reaching important but uneasy goals. Several studies have demonstrated the influence of ZTPI on psychological well-being, cf. [3] and [4]. ZTPI-based therapies, which imply stepwise changes of the corresponding time perspectives show success even in cases of severe psychological illnesses like in the case of Post-Traumatic-Stress-Disorder, or PTSD [5]. A German study has shown that time perspective could influence the burnout tendency of students [6]. Essential for wellbeing is the concept of Balanced Time Perspectives—or BTP [7]; [8]; [4]; [9]; [10] and [11] respectively the concept of Deviation Balanced Time Perspectives (DBTP), which was developed by [12]. For an overview compare also [13]. According to [12], a balanced time perspective is characterized by the following configuration of the five dimensions: a) PP highly pronounced, b) NP weakly pronounced, c) HP moderately pronounced, d) FP weakly pronounced and, f) F moderately pronounced. Further, the influence on dysfunctional or problematic health or social behaviors such as risky driving [14], addictive behavior [15] as well as risk-taking [16] was in the scope of research on time perspectives. A recent study also shows a high relevance of time perspectives for the acceptance of unethical behavior in the context of business ethics [17]. In summary, research in the last ten years has shown that time orientation is highly relevant for a wide range of domains of human life related to success and well-being. According to management and working behavior it seemed also highly relevant, especially according to self-control and achievement at the working place. According to achievement behavior, which may be most relevant for management issues, we can summarize that a high Future orientation maybe most relevant. Further, one could assume the general well-being of person mediates the influence of BTP or DBTP (Deviation from Balanced Time Perspectives) on achievement.

\section{The Hofstede Dimensions (HD)}

When talking about culture, we notice that literature review corpus gives us many different understandings and definitions of the concept. Reference [18] defines culture as a set of patterns and ways of thinking, feeling, and reacting, acquired and transmitted mainly by symbols. Those patterns constitute the distinctive achievements and characteristics of human groups. Reference [19] conceived culture as a construct that manifests itself in an organization as a result of the organization's location within a particular society. Those two definitions lead us to introduce the concept of national culture. Hofstede's study of the national cultural dimensions, published in 1980, was the first study to compare countries using cultural dimensions, which included more than 50 countries. Over several decades, this study has been best known as a cross-cultural study. Despite the criticisms addressed to the Hofstede model (cf. for overview [20] and [21]), the Hofstede dimensions (cf. [19] and [22]) are part of a widespread concept to measure and assess cultural differences. Central is the assumption of several cultural dimensions. In the first version, five dimensions were assumed: 1) individualism vs. collectivism (IDV), 2) power distance (PDI), 3) masculinity (MAS), and 4) uncertainty avoidance (UAI). The model was enlarged stepwise by adding three further dimensions: 5) long-term vs. short-term orientation (LTO) [23], 6) indulgence vs. restraint (IDV) [24], 7) monumentalism vs. flexhumity (MON), and 8) exclusionism vs. universalism [25]. We assume that some of the Hofstede dimensions are related to time perspective orientation.

\section{Research Model and Hypothesis}

\subsection{Relationship between HD and ZTPI}

Our research model consists of the Hofstede cultural dimensions at the individual level as dependent variables. It is assumed that the time perspectives have an influence on how strong some of the Hofstede cultural dimensions will be pronounced at the individual level. The time perspectives were assumed as independent variables. The hypotheses were tested in three cultural settings: Germany, Morocco, and China. One has to consider that the model assumes a measurement on the group level. We, however, analyze the relationship between both constructs at the individual level. This is discussable (for discussion, problems and results cf. [26]; [27] and [28]) but it seems reasonable because we have to measure the individual variation of the Hofstede dimensions to test how it is influenced by time perspectives. We assumed that the following cultural dimensions will be influenced 
by time perspectives: LTO, UAI and IVR. According to LTO, we assume a positive influence of Future (high Future orientation will result in long-term-orientation) and a negative influence by Positive Past (in the Hofstede-model short-term orientation is based on relying on traditional values of a society as also emphasized by individuals scoring high at Positive Past time perspective). We assume that Future, Negative Past and Fatalistic Present time perspective will enhance uncertainty-avoidance tendencies. The first factor, because those individuals, who think more about the Future, will also try more, to avoid uncertainty. Persons with high Negative Past and high Fatalistic Present orientation become more unsettled and thus show stronger attempts to avoid uncertainty. A high Positive Past orientations, has an inverse effect my reducing the subjective uncertainty and thus, create higher tolerance towards uncertainty. According to IVR we assume that those with high Hedonistic Present orientation will perceive the restraints by the society as stronger and will thus show higher IVR values at the individual level (cf. for all possible relationships Figure 1).

\subsection{Summary of Hypotheses}

H1: High Future orientation will increase LTO*.

H2: High Positive Past orientation will decrease LTO*;

H3: High Future orientation will increase UAI*

H4: High Fatalistic Present will increase UAI*.

H5: High Negative Past will increase UAI*.

H6: High Positive Past will decrease UAI*.

H7: High Hedonistic will increase IVR*.

("All cultural dimensions were measured at the individual level).

\subsection{Methodology}

The Hofstede dimensions were measured by the VSM 08, and for measuring the time perspectives, we used the German version by [29] for Germany and the validated French version by [30] for Morocco. In the case of China, we translated the items of the ZTPI into Chinese through bilingual Chinese translators, which were also carefully checked by other bilingual translators. The sample sizes were $n=193$ (Germany), $n=209$ (Morocco), and $n=192$ (China). All participants were regular students. Those who took part in the survey study were students of the University of Applied Sciences, Heilbronn (Germany); students of the School of Management, Fuzhou (China); and students of the ENCG, Casablanca (Morocco). For analysis, we used linear regressions.

\section{Results}

\subsection{Long-Term-Orientation (LTO)}

According to LTO we confirmed our hypotheses of the enhancing influence of Future (H1) and the reducing ef-

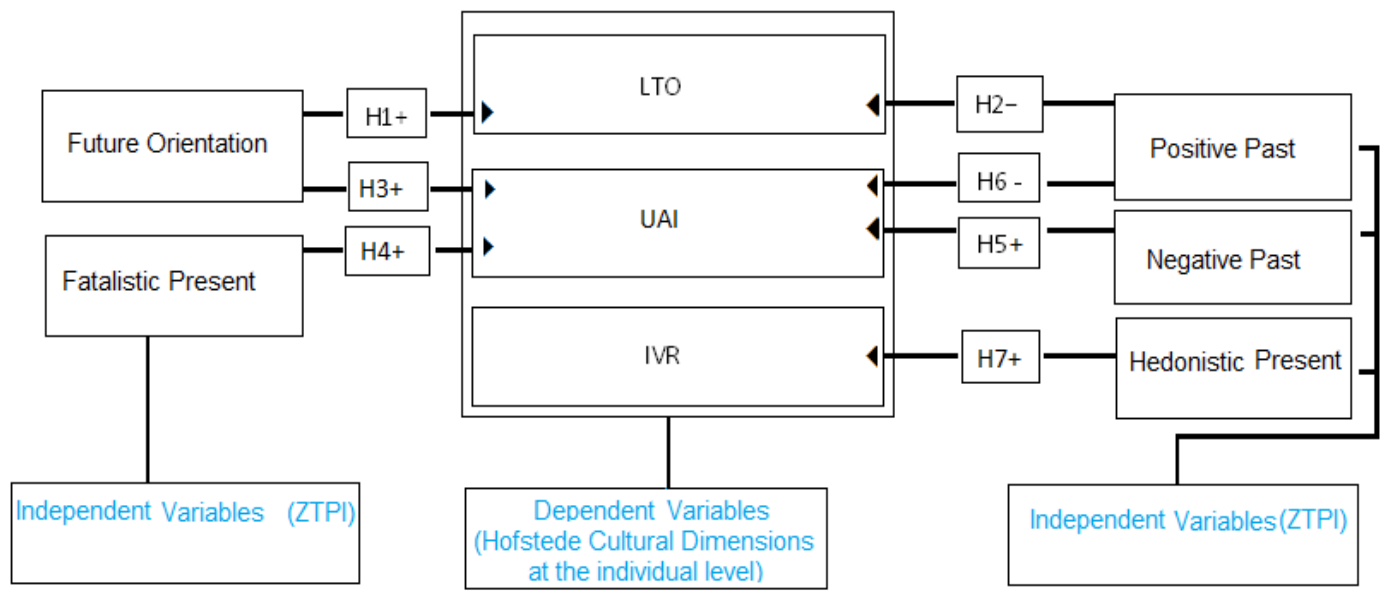

Figure 1. Possible relationshio between ZTPI and cultural dimensions by Hofstede-model. 
fect of Positive Past (H2) in Morocco (for Future: $\beta=0.21, t(204)=2.84, p=0.005$; for Positive Past: $\beta=-0.23$, $t(204)=-3.14, p=0.002$ ) and in Germany (for Future: $\beta=0.15, t(183)=1.95, p=0.053$; for Positive Past: $\beta=$ $-0.21, t(183)=-2.80, p=0.006$, whereas, both effects did not reach significance in China (all $\beta$ 's $<0.04$, all t’s $(188)<0.48$, all $p$ 's $>0.635$.).

\subsection{Uncertainty-Avoidance-Index (UAI)}

The hypotheses according UAI (H3, H4, H5 and H6) were confirmed in the same way for the Moroccan (for Future: $\beta=0.11, t(197)=1.71, p=0.089$; for Fatalistic Present: $\beta=0.33, t(197)=4.32, p=0.000$; for Negative Past: $\beta=0.19, t(197)=2.60, p=0.010$; Positive Past: $\beta=-0.16, t(197)=-2.48, p=0.014$ ) and for the German subsample (for Future: $\beta=0.35, t(182)=4.90, p=0.000$; for Fatalistic Present: $\beta=0.19, t(182)=2.31, p=$ 0.022; for Negative Past: $\beta=0.17, t(182)=2.18, p=0.030$; Positive Past: $\beta=-0.33, t(182)=-4.32, p=0.000)$. Again, however we observed contradictory non-supporting results for the Chinese subsample. Only the enhancing effect of Negative Past (H5) was observed for China at the 10\%-level, $\beta=0.16, t(186)=1.77, p=0.070$, but all other factors were non-significant, all $\beta$ 's $<0.06$, all $t$ 's(186) $<0.57$, all $p$ 's $>0.573$.

\subsection{Indulgence versus Restraint (IVR)}

For Morocco Hedonistic Present (H7) reached significance at the 10\%-level, $\beta=0.12, t(207)=1.70, p=0.091$ and for Germany significance at the 5\%-level, $\beta=0.23, t(182)=3.23, p=0.001$, whereas again the results for China showed a differing patter: The factor Hedonistic Present reached no significance, $\beta<-0.09, t(189)<$ $-1.43, p>0.151$.

\section{Discussion}

The first essential limitation is the use of student samples in all three analysed cultures, which has to be considered as a limitation in so far as one could not derive general statements about the general population in the three considered cultures-Morocco, China, and Germany. A limitation on students may seem reasonable in two essential respects: First, due to practical reasons. It is much easier to collect survey data from students properly than from any other group within a society. Second, the use of students allows a comparison across cultures, because potential relevant disturbance variables — such as age, educational level, to some degree also income and lifestyle orientation, and others - could be held constant and thus controlled. The second limitation is the fact that the used ZTPI scales were not yet validated in the Chinese and German languages (whereas the used French version was already validated). Several significant relationships between ZTPI and Hofstede dimensions at the individual level were observed. These effects were, however, not entirely consistent across the three cultural settings. In two-Morocco and Germany — out of three cultural settings supporting results were observed, whereas contradictory mostly non-significant results were observed for China. Thus further theoretical considerations and empirical studies are necessary to explain these differences. Future research has to clarify if the deviant results in China are based on measurement biases or on existing differences. The supporting results for both other samples-Morocco and Germany — could be evaluated as preliminary confirmation of our theoretical model, but further studies are necessary. Future research should also include-beside ZTPI and Hofstede dimensions at the individual level — the Hofstede dimensions at the level of the society and clarify their role according to ZTPI and Hofstede dimensions at the individual level.

\section{Acknowledgements}

We like to thank to Prof. Dr. Mohammed Nabil Benchekroun (Directeur de ENSG, Casablanca), Prof. Dr. Peter Mudra (President of the University of Applied Sciences Ludwigshafen), Prof. Dr. Rainer Busch (Vice-President of the University of Applied Sciences Ludwigshafen), and Prof Dr. Zhengpeng Tang (Dean of the School of Management, Fuzhou University) for their organizational support in enabling this research project.

\section{References}

[1] Zimbardo, P.G. and Boyd, J.N. (1999) Putting Time into Perspective. A Valid, Reliable Individual-differences Metric Measurement. Journal of Personality and Social Psychology, 77, 1271-1288.

http://dx.doi.org/10.1037/0022-3514.77.6.1271 
[2] Boyd, J.N. and Zimbardo, P.G. (2005) Time Perspective, Health, and Risk Taking. In: Strathman, A. and Joireman, J., Eds., Understanding Behavior in the Context of Time: Theory, Research, and Applications, Erlbaum, Mahwah, 85-107.

[3] Brown, K.W. and Ryan, R.M. (2003) The Benefits of Being Present: Mindfulness and Its Role in Psychological Well-Being. Journal of Personality and Social Psychology, 84, 822-848. http://dx.doi.org/10.1037/0022-3514.84.4.822

[4] Drake, L., Duncan, E., Sutherland, F., Abernethy, C. and Henry, C. (2008) Time Perspective and Correlates of Wellbeing. Time \& Society, 17, 47-61. http://dx.doi.org/10.1177/0961463X07086304

[5] Zimbardo, P.G., Sword, R. and Sword, R. (2012) The Time Cure: Overcoming PTSD with the New Psychology of Time Perspective Therapy. Jossey-Bass, San Francisco.

[6] Unger, A. (2013) Influence of Time Perspectives on the Affective Component of Burnout Tendency. Conference Paper presented at the 5th Asian Conference of Health Psychology (ACHP), Daejeon, 22-24 August, 82.

[7] Boniwell, I., Osin, E., Linley, P. and Ivanchenko, G. (2010) A Question of Balance: Time Perspective and Well-being in British and Russian Samples. The Journal of Positive Psychology, 5, 24-40. http://dx.doi.org/10.1080/17439760903271181

[8] Boniwell, I. and Zimbardo, P.G. (2004) Balancing Time Perspective in Pursuit of Optimal Functioning. In: P. Alex, Linley and Stephan Joseph, Eds., Positive Psychology in Practice, John Wiley \& Sons, Hoboken, 165-178.

[9] Ivanchenko, G., Osin, E. and Leontiev, D. (2009) Perception of Possibilities, Subjective Well-Being and Future Time Perspective. Poster Presented at the First World Congress on Positive Psychology, Philadelphia, 18-21 June.

[10] Osin, E., Boniwell, I., Linley, P.A. and Ivanchenko, G. (2009) Balanced Time Perspective in Britain and in Russia. Conference Paper Presented at the First World Congress on Positive Psychology, Philadelphia, 18-21 June.

[11] Webster, J.D. (2011) A New Measure of Time Perspective: Initial Psychometric Findings for the Balanced Time Perspective Scale (BTPS). Canadian Journal of Behavioural Science/Revue Canadienne des Sciences du Comportement, 43, 111-118.

[12] Stolarski, M., Bitner, J. and Zimbardo, P.G. (2011) Time Perspective, Emotional Intelligence and Discounting of Delayed Awards. Time \& Society, 20, 346-363. http://dx.doi.org/10.1177/0961463X11414296

[13] Zhang, J., Howell, R. and Stolarski, M. (2013) Comparing Three Methods to Measure a Balanced Time Perspective: The Relationship Between a Balanced Time Perspective and Subjective Wellbeing. Journal of Happiness Studies, 14, 169-184. http://dx.doi.org/10.1007/s10902-012-9322-x

[14] Zimbardo, P.G., Keough, K.A. and Boyd, J.N. (1997) Present Time Perspective as a Predictor of Risky Driving. Personality and Individual Differences, 23, 1007-1023. http://dx.doi.org/10.1016/S0191-8869(97)00113-X

[15] Keough, K.A., Zimbardo, P.G. and Boyd, J.N. (1999) Who’s Smoking, Drinking and Using Drugs? Time Perspective as a Predictor of Substance Use. Basic and Applied Social Psychology, 21, 149-164. http://dx.doi.org/10.1207/S15324834BA210207

[16] Boyd, J.N. and Zimbardo, P.G. (1997) Constructing Time after Death: The Transcendental-Future Time Perspective. Time \& Society, 6, 35-54. http://dx.doi.org/10.1177/0961463X97006001002

[17] Unger, A., Busch, R., Hau, L.-N. and Wang, Y.-C. (2012) The Influence of Time Perspectives (ZTPI) on the Acceptance of Unethical Behavior in the Context of Business Ethics-A Cross-Cultural Comparison between China, Vietnam and Germany. Conference Paper Presented at the 1th International Conference on Time Perspectives (ICTP), Coimbra, 5-8 September, 62-63.

[18] Kluckhohn, C. (1951) Values and Value-Orientations in the Theory of Action: An Exploration in Definition and Classification. In: Parsons, T. and Shils, E., Eds., Toward a General Theory of Action, Harvard University Press, Cambridge, 388-433. http://dx.doi.org/10.4159/harvard.9780674863507.c8

[19] Hofstede, G. (1980) Culture’s Consequences_-International Differences in Work Related Values. Sage, Newbury Park.

[20] House, R.J., Wright, N.S. and Aditya, R.N. (1997) Cross-Cultural Research on Organizational Leadership: Measurement of Cultural Dimensions. In: Earley, P.C. and Erez, M., Eds., New Perspectives on International Industrial/Organizational Psychology, New Lexington Press, San Francisco, 571-581.

[21] McSweeney, B. (2002) Hofstede's Model of National Cultural Differences and their Consequences: A Triumph of Faith-A Failure of Analysis. Human Relations, 55, 89-118. http://dx.doi.org/10.1177/0018726702055001602

[22] Hofstede, G. and Hofstede, G.J. (2005) Culture and Organizations-Software of the Mind: Intercultural Cooperation and its Importance for Survival. 2nd Edition, McGraw Hill, New York.

[23] Hofstede, G. (2001) Culture’s Consequences-Comparing Values, Behaviors, Institutions and Organizations across Nations. 2nd Edition, Sage, Thousand Oaks.

[24] Hofstede, G., Hofstede, G.J. and Minkov, M. (2010) Cultures and Organizations: Software of the Mind. McGraw-Hill, New York.

[25] Minkov, M. and Hofstede, G. (2012) Hofstede’s Fifth Dimension: New Evidence from the World Values Survey. 
Journal of Cross-Cultural Psychology, 43, 2-14. http://dx.doi.org/10.1177/0022022110388567

[26] Fischer, R., Vauclair, C.-M., Fontaine, J.R.J. and Schwartz, S.H. (2010) Are Individual-Level and Country-Level Value Structures Different? Testing Hofstede's Legacy with the Schwartz Value Survey. Journal of Cross-Cultural Psychology, 41, 135-151. http://dx.doi.org/10.1177/0022022109354377

[27] Søndergard, M. (1994) Research Note: Hofstede’s Consequences: A Study of Reviews, Citations and Replications. Organization Studies, 15, 447-456. http://dx.doi.org/10.1177/017084069401500307

[28] Yoo, B., Donthu, N. and Lenartowicz, T. (2011) Measuring Hofstede's Five Dimensions of Cultural Values at the Individual Level: Development and Validation of CVSCALE. Journal of International Consumer Marketing, 23, 193210.

[29] Milfont, T.L. (2008) Zimbardo Time Perspective Inventory Germany.

[30] Apostolidis, T. and Fieulaine, N. (2004) French Validation of the Zimbardo Time Perspective Inventory. European Review of Applied Psychology, 54, 207-217. http://dx.doi.org/10.1016/j.erap.2004.03.001 